\title{
EVN observation of three Broad Absorption Line quasars
}

\author{
D. R. Jiang ${ }^{1}$ and T. G. Wang ${ }^{2}$ \\ 1 Shanghai Astronomical Observatory, Chinese Academy of Sciences, Shanghai 200030, PR China \\ 2 Center for Astrophysics, University of Science and Technology of China, Hefei, Anhui 230026, PR China \\ Received 8 November 2002 / Accepted 1 December 2002
}

\begin{abstract}
We present the results of European VLBI Network observations of 3 Broad Absorption Line quasars at 1.6 GHz. They are all compact with linear sizes less than $1 \mathrm{kpc}$. The flat spectral source, J1556+3517, is unresolved at about 20 mas resolution, indicating that the orientation of the radio jet in this source is probably near the line of sight. J1312+2319 shows asymmetric two-sided structure on a scale of several hundred pc. This structure resembles that of Compact Symmetric Objects (CSO), however it has a bright central component at the present resolution. The jet orientation of J1312+2319 may be far from the line of sight. J0957+2356 is also unresolved, but with a steep spectrum, therefore the jet orientation in this source is unclear. These results are not consistent with the unification of BAL and non-BAL quasars by orientation.
\end{abstract}

Key words. galaxies: active - galaxies: jets - quasars: absorption lines - quasars: general

\section{Introduction}

About $10 \%$ of optically selected quasars display broad absorption troughs, which extend up to $0.1 \mathrm{c}$ blueward of the high ionization resonant lines (Weymann et al. 1991). Ten percent of them also show absorption troughs in low ionization lines. The high ionization level, and continuous absorption over a large velocity range indicates that the absorption lines are intrinsic to the nucleus. The blueshifting of lines implies that the absorbing gas is flowing outward from the nucleus.

A general paradigm has been established that Broad Absorption Line (BAL) regions exist in both BAL and nonBAL quasars, and that BAL quasars are normal quasars seen along a particular line of sight. The spectropolarimetry results can be explained by an equatorial BAL region which intersects the line of sight in a BAL quasar (Goodrich \& Miller 1995; Hines \& Wills 1995; Cohen et al. 1995). Hence, the absorbing material could be a wind which is accelerated by radiation pressure from an accretion disk or torus, and BAL quasars are seen at large inclination.

The BAL quasars were found preferentially in radio quiet quasars, and were not found as radio loud until the NRAO VLA Sky Survey (NVSS) and the FIRST deep radio survey were carried out. The FIRST survey detected more than 40 radio BAL quasars, most of which can be formally classified as radio-loud quasars based on the radio to optical flux ratio as well as on the radio power (Becker et al. 2000; Menou et al. 2001). Brotherton et al. (1998) discovered 5 radio-loud BAL quasars detected in the NVSS. Most of the BAL quasars in Becker et al.'s sample are unresolved at VLA resolutions with either a flat or a steep radio spectrum.

Send offprint requests to: D. R. Jiang, e-mail: djiang@center.shao.ac.cn
The radio morphology of the BAL quasars is very important in distinguishing between various models for BAL quasars. The radio morphology can serve as an inclination indicator of the BAL quasars, and therefore yields a direct test of the orientation model. It is generally expected that the axis of the accretion disk will be aligned with the radio jets. If the BAL region is equatorial, we would expect to see two side jets in these objects.

But unfortunately, we have very limited information about the radio structure of the BAL quasars. Only three BAL quasars, FIRST J101614.3+520916 (Gregg et al. 2000), PKS 1004+13 (Wills et al. 2001; Kellermann et al. 1994), and LBQS 1138-0126 (Brotherton et al. 2002) are known as double-lobed radio morphology on kpc scales. The weak core, strong lobe emissions, and symmetric morphology make these sources "edge-on" in the unification scheme. The relatively steep spectrum $(\alpha=-0.63)$ of the radio core of J1016+5209 suggests that it may be a Compact Steep Spectrum (CSS) source, but its large scale FRII radio lobes do not fit the picture of it being young (Gregg et al. 2000).

Based on the large scatter in radio spectral indices, Becker et al. (2000) argued that BAL quasars are not viewed at any particular orientation. An alternative picture is that BAL quasars are an early stage in the development of new or refuelled quasars. Although the radio spectrum is usually a good indicator of the radio jet inclination, it is not well known whether this applies to the BAL quasars, as the radio jet in these objects might be more compact. The high resolution images are imperative.

In this paper we present EVN observations of three BAL quasars at $1.6 \mathrm{GHz}$ and some discussion about these results. We adopt the value of $H_{0}=65 \mathrm{~km} \mathrm{~s}^{-1} \mathrm{Mpc}^{-1}$ for the Hubble constant and $q_{0}=0.5$ for the deceleration parameter, assuming 
the cosmological constant $\Lambda=0$; and a spectral index definition $S_{v} \propto v^{\alpha}$ is used in this paper.

\section{Observation and data reduction}

The observation of 3 BAL quasars, FBQS J095707.3+235625 (J0957+2356), FBQS J131213.5+231958 (J1312+2319) and FIRST J155633.8+351758 (J1556+3517), at $1.6 \mathrm{GHz}$ was carried out with the European VLBI Network (EVN) on 2001 May 26. The left-circular polarization signals were recorded in 8 intermediate frequency (IF) bands with a total bandwidth of $32 \mathrm{MHz}$ and 2 bit sampling. The phase referencing sources were J0956+2515, J1321+2216, and J1602+3326 respectively, of which the angular distances from the target sources are less than $2^{\circ} .4$. The scan time on each BAL quasar was about $170 \mathrm{~min}$. The data were correlated at the Joint Institute for VLBI in Europe (JIVE) in Dwingeloo. The Urumqi station failed during the observation. As a result, there is a big gap between the European baselines and the baselines related to the Shanghai station. The EVN pipeline results were used for the phase referencing, and the sources were strong enough such that the normal fringe fitting also detected these sources. The results of the phase referencing and non-phase referencing are in good agreement. The initial amplitude calibration and the fringe-fitting were performed using the NRAO Astronomical Image Processing System (AIPS). The imaging and self-calibration were carried out in DIFMAP (Shepherd et al. 1994) and these self-calibrations allowed us to improve the ratio of signal to noise in the images. The estimated uncertainty of the amplitude calibration is about 10 percent.

\section{Sources and results}

$\mathrm{J} 1556+3517$ was discovered as a low-ionization BAL quasar at a redshift of $z=1.48$ with a heavily absorbed continuum by Becker et al. (1997). It is a point source with $30.6 \mathrm{mJy}$ in the FIRST catalog, and the flux density in the Green Bank $5 \mathrm{GHz}$ catalog is $27 \mathrm{mJy}$. These non-simultaneous data suggest a flat spectral index $\alpha=-0.1$. The radio loudness with no $K$-correction is $\log R>3$ (Becker et al. 1997), while Najita et al. (2000) gave a low value $\log R \approx 0.9$ after extinction correction and $K$-correction. The naturally weighted image with the European antennas only (Fig. 1) shows a compact component with a 27 mJy flux density.

$\mathrm{J} 1312+2319$ is an HiBAL quasar at a redshift of $z=1.508$ (1 mas corresponds to $4.1 \mathrm{pc}$ at this redshift). The VLA observations in the different configurations at $20 \mathrm{~cm}$ suggest that the source is unresolved with a flux density of about $46 \mathrm{mJy}$, the spectral index between $20 \mathrm{~cm}$ and $3.6 \mathrm{~cm}$ is $\alpha=-0.8$, and the estimated radio loudness is $\log R=1.88$ (Becker et al. 2000). The naturally weighted image with the European antennas only (Fig. 2) shows an asymmetric two-sided structure. This two-sided structure is not well aligned with the central component. The south-western jet is stronger than the northern one. The total flux density of the clean components is $40 \mathrm{mJy}$, and the flux density of the central component is about $31 \mathrm{mJy}$, i.e. about $80 \%$ of total flux density. The most distant component

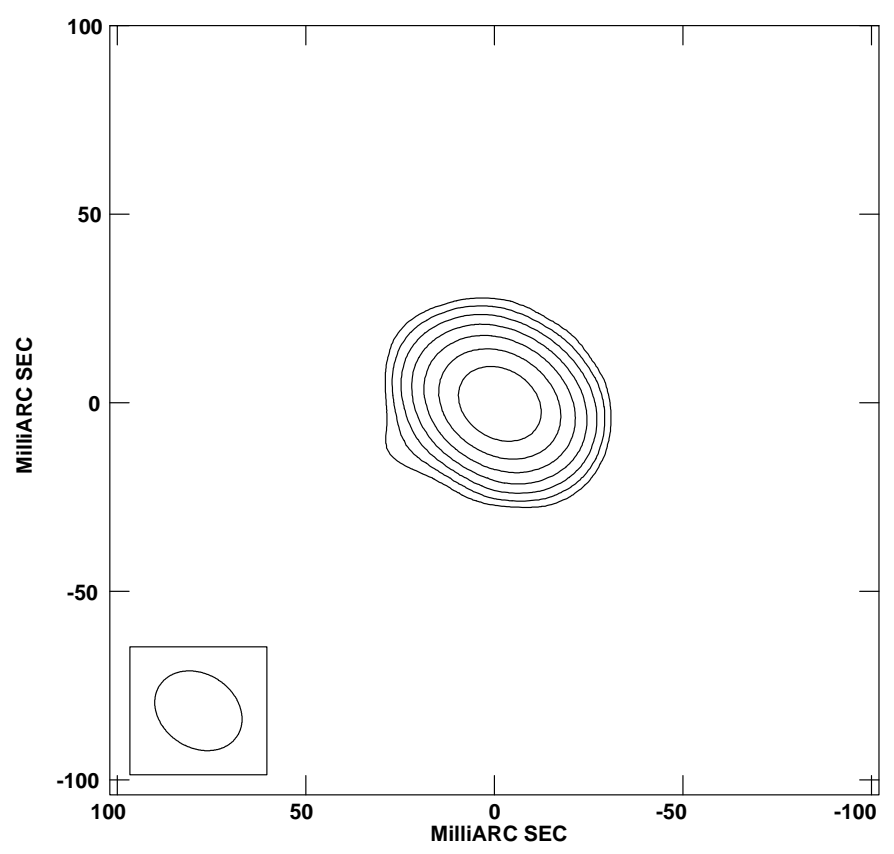

Fig. 1. EVN image with the European antennas only of J1556+3517 at $1.6 \mathrm{GHz}$. The restoring beam is $24.6 \times 19.5$ mas at $\mathrm{PA}=56.6$, the contour levels are $(-1,1,2,4,8,16,32,64,128) \times 0.22 \mathrm{mJy} / \mathrm{beam}$, and the peak flux density is $25.9 \mathrm{mJy} / \mathrm{beam}$.

is about 60 mas (corresponding to about $240 \mathrm{pc}$ ) away from the central component.

HiBAL J0957+2356 $(z=1.995)$ is the most luminous source at radio wavelengths in Becker et al.'s catalog with a radio loudness $\log R=2.63$. The flux densities at $20 \mathrm{~cm}$ and $3.6 \mathrm{~cm}$ measured by the VLA were about $139 \mathrm{mJy}$ and $51.3 \mathrm{mJy}$ respectively, which suggests a spectral index of $\alpha=-0.6$ (Becker et al. 2000). The source shows an unresolved component with a flux density of about $136 \mathrm{mJy}$ at $18 \mathrm{~cm}$ with about 20 mas resolution (Fig. 3). Figure 4 is the naturally weighted image including the Shanghai station's data. The source is barely resolved at the longest baselines.

\section{Discussion}

The lower limits of the brightness temperature in the source rest frame of the strongest component in these BALs, based on the results of model-fitting, are higher than $1.3 \times 10^{9} \mathrm{~K}$. This high brightness temperature suggests that the radio emission comes from the synchrotron emission of the jet.

The flux density of the compact component at $18 \mathrm{~cm}$ in $\mathrm{J} 1556+3517$ is very close to the flux density measured by the VLA at $20 \mathrm{~cm}$. If the compact component is the base of the jet, this source could be core-dominated at $18 \mathrm{~cm}$. The compact structure is consistent with its flat spectrum, and therefore the radio jet orientation in this BAL quasar might be near the line of sight.

But in $\mathrm{J} 0957+2356$, the flux density of the compact component is $136 \mathrm{mJy}$ at $18 \mathrm{~cm}$, the flux density at $3.6 \mathrm{~cm}$ measured by the VLA in D configuration is $51.3 \mathrm{mJy}$ (Becker et al. 2000). Assuming that all the emission at $3.6 \mathrm{~cm}$ comes from the compact component and that the source is invariable, the core 


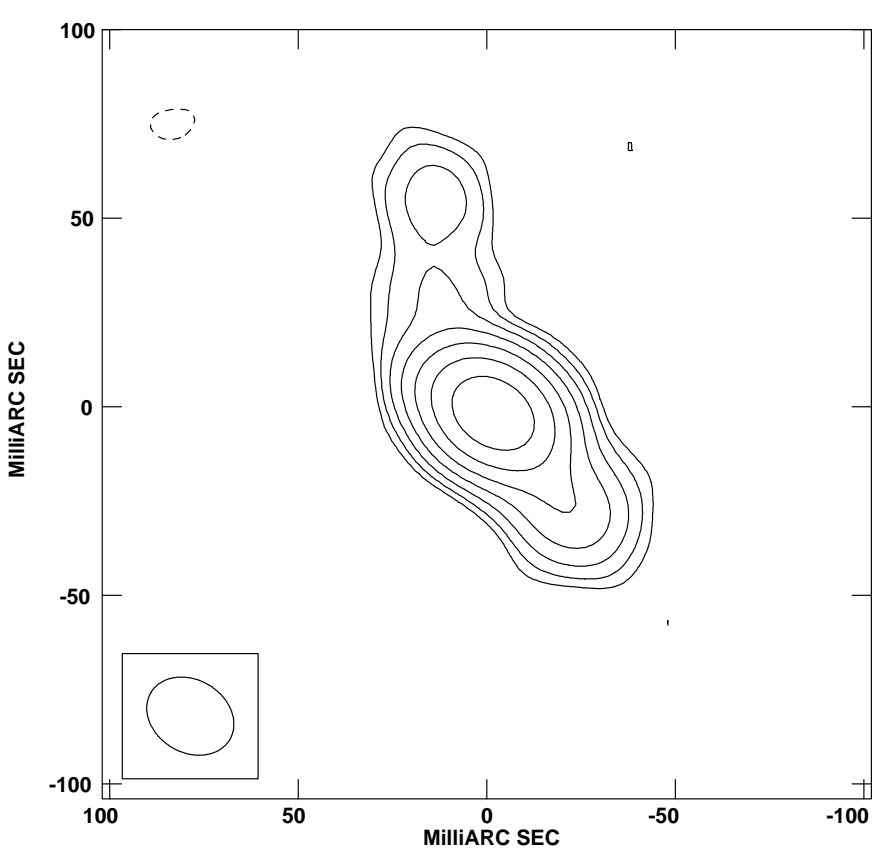

Fig. 2. EVN image with the European antennas only of J1312+2319 at $1.6 \mathrm{GHz}$. The restoring beam is $24.4 \times 19.2$ mas at $\mathrm{PA}=59^{\circ}$, the contour levels are $(-1,1,2,4,8,16,32,64) \times 0.28 \mathrm{mJy} / \mathrm{beam}$, and the peak flux density is $30.9 \mathrm{mJy} / \mathrm{beam}$.

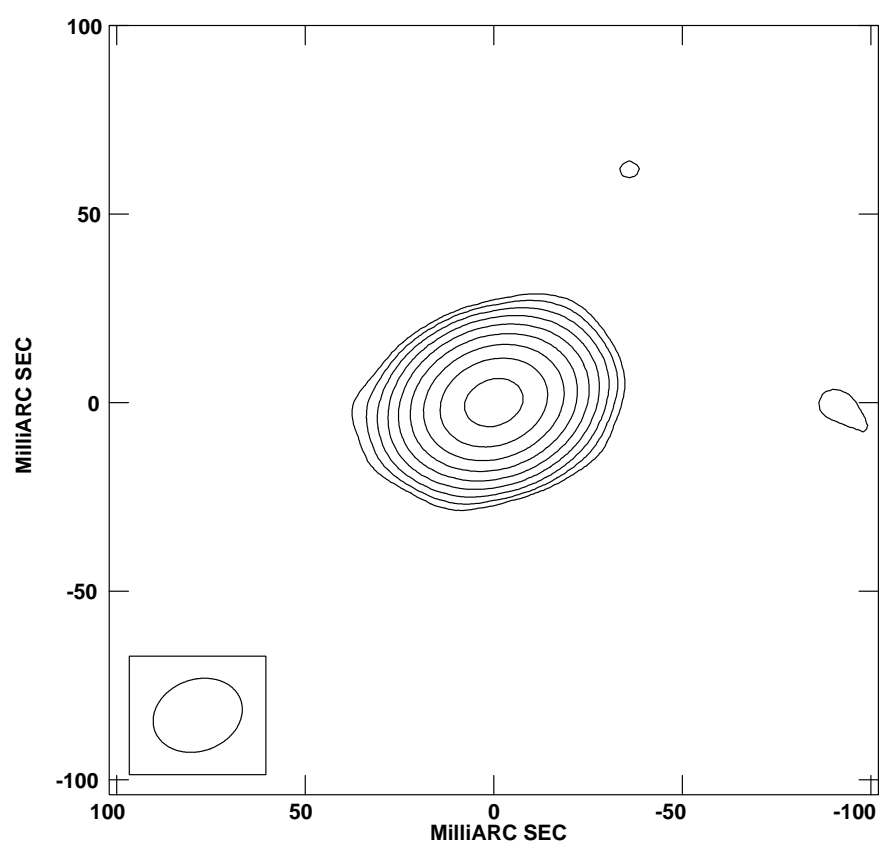

Fig. 3. EVN image with the European antennas only of J0957+2356 at $1.6 \mathrm{GHz}$. The restoring beam is $24.2 \times 19$ mas at $\mathrm{PA}=-70^{\circ}$, the contour levels are $(-1,1,2,4,8,16,32,64,128,256) \times 0.4 \mathrm{mJy} / \mathrm{beam}$, and the peak flux density is $134 \mathrm{mJy} / \mathrm{beam}$.

has a steep spectrum with $\alpha=-0.6$. Therefore, it is likely that $\mathrm{J} 0957+2356$ is a CSS source although the source size is much smaller than the typical value for a CSS source. It is quite likely that the jet in this source is intrinsically very short, rather than short due to projection effects. Higher resolution observations will allow us to test this hypothesis, and simultaneous observations at different frequencies will allow us to confirm or

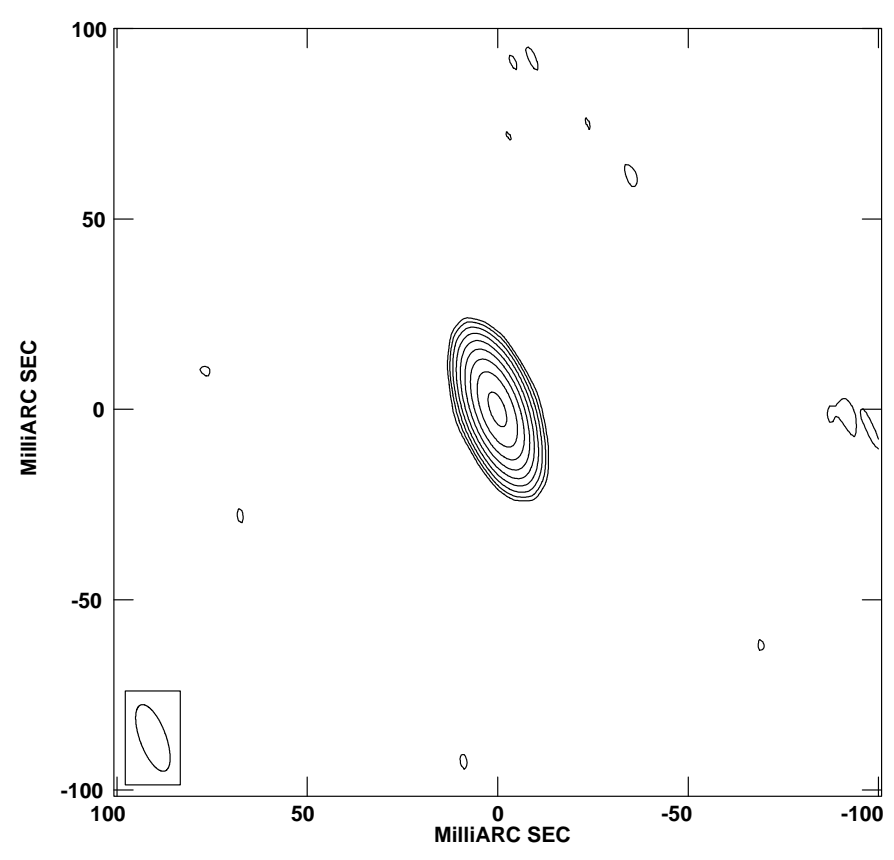

Fig. 4. EVN full resolution image of J0957+2356 at $1.6 \mathrm{GHz}$. The restoring beam is $18.5 \times 6.86$ mas at $\mathrm{PA}=20^{\circ} 1$, the contour levels are $(-1,1,2,4,8,16,32,64,128,256) \times 0.4 \mathrm{mJy} /$ beam, and the peak flux density is $124 \mathrm{mJy} /$ beam.

reject the possibility that the steep spectrum of the core is real and not due to source variability.

The two-sided structure in J1312+2319 suggests that the orientation of the jet is far from the line of sight. The source size is only several hundred pc, the same as the typical size of a Compact Symmetric Object (CSO), but the flux density of the central component is about $80 \%$ of the total flux density, which is different from the structure of CSOs. The core of CSOs, in general, is weak at low frequency. The asymmetric two-sided morphology in $\mathrm{J} 1312+2319$ suggests that the absorbing gas probably lies close to the equatorial plan, but the bright central component is inconsistent with this explanation and further observations at higher resolution are necessary to further understanding this structure.

\section{Summary}

EVN observations at $18 \mathrm{~cm}$ of 3 radio-loud BAL quasars have revealed their different radio morphology. $\mathrm{J} 1556+3517$ is a point-like source at about 20 mas resolution. The core dominated structure is consistent with its flat spectrum. Therefore, the orientation of the jet in this source is probably near the line of sight. J0957+2356 is barely resolved at the longest baseline. With a steep radio spectrum, this radio source may be intrinsically smaller than our angular resolution, rather than small due to projection effects. Thus, the jet orientation is unclear in this source. J1312+2319 has an asymmetric two-sided structure. The jet orientation is quite likely far from the line of sight. Like a CSO, the source extends only several hundred pc, but in contrast has a bright central component at the present resolution. Further high resolution observations will help us to clarify if the source can be classified as a CSO. Although the number 
of sources is still too small to make any firm statistical conclusions, it would seem that the unification of BAL and non-BAL quasars by orientation may be problematic at least for these sources.

Acknowledgements. This work was supported by the Chinese fund NKBRSF(G1999075403). TW acknowledges the financial support from the Chinese NSF-19925313. The European VLBI Network is a joint facility of European, Chinese, and other radio astronomy institutes funded by their national research councils.

\section{References}

Becker, R. H., Gregg, M. D., Hook, I. M., et al. 1997, ApJ, 479, L93 Becker, R. H., White, R. L., Gregg, M. D., et al. 2000, ApJ, 538, 72
Brotherton, M. S., van Breugel, W., Smith, R. J., et al. 1998, ApJ, 505, L7

Brotherton, M. S., Croom, S. M., Breuck, C. D., et al. 2002, AJ, 124, 2575

Cohen, M. H., Ogle, P. M., Tran, H. D., et al. 1995, ApJ, 448, L77

Goodrich, R. W., \& Miller, J. S. 1995, ApJ, 448, L73

Gregg, M. D., Becker, R. H., Brotherton, M. S., et al. 2000, ApJ, 544, 142

Hines, D. H., \& Wills, B. J. 1995, ApJ, 448, L69

Kellermann, K. I., Sramek, R. A., Schmidt, M., et al. 1994, AJ, 108, 1163

Menou, K., Berk, D. E. V., Ivezić, Z̆., et al. 2001, ApJ, 561, 645

Najita, J., Dey, A., \& Brotherton, M. 2000, AJ, 120, 2859

Shepherd, M. C., Pearson, T. J., \& Taylor, G. B. 1994, BAAS, 26, 987

Wills, B. J., Brandt, W. N., \& Laor, A. 1999, ApJ, 520, L91

Weymann, R. J., Morris, S. L., Foltz, C. B., et al. 1991, ApJ, 373, 23 